\title{
An Extended R-Tree Indexing Method Using Selective Prefetching in Main Memory
}

\author{
Hong-Koo Kang, Joung-Joon Kim, Dong-Oh Kim, and Ki-Joon Han \\ School of Computer Science \& Engineering, Konkuk University, \\ 1, Hwayang-Dong, Gwangjin-Gu, Seoul 143-701, Korea \\ \{hkkang, jjkim9, dokim, kjhan\}@ab.konkuk.ac.kr
}

\begin{abstract}
Recently, researches have been performed on a general method that can improve the cache performance of the R-Tree in the main memory to reduce the size of an entry so that a node can store more entries. However, this method generally requires additional processes to reduce information of entries. In addition, the cache miss always occurs on moving between a parent node and a child node. To solve these problems, this paper proposes the SPR-Tree (Selective Prefetching R-Tree), which is an extended R-Tree indexing method using selective prefetching according to node size in the main memory. The SPR-Tree can produce wider nodes to optimize prefetching without additional modifications on the R-Tree. Moreover, the SPR-Tree can reduce the cache miss that can occur in the R-Tree. In our simulation, the search, insert, and delete performance of the SPR-Tree improved up to $40 \%, 10 \%, 30 \%$ respectively, compared with the R-Tree.
\end{abstract}

Keywords: SPR-Tree, Extended R-Tree, Cache Performance, Cache Miss, Main Memory.

\section{Introduction}

Recently, with the speed gap being broader between the processor and the main memory, how effectively to use the cache memory in the main memory-based index is making a critical impact on the performance of the entire system[1,5]. The R-Tree is similar to the B-Tree, but is used for spatial access methods for indexing multidimensional data[2]. Since the R-Tree is originally designed to reduce disk I/O effectively for the disk-based index, the node size is optimized for disk block.

However, the R-Tree is not suitable for the cache memory with a small block. Delay time caused by cache miss accounts for a significant part of the entire performance time[10]. Especially, when the R-Tree, as in a main memory DBMS, resides in the main memory, disk I/O does not affects the entire performance seriously. Consequently, studies on the index structure and algorithms with the improved cache performance are being carried out by numerous researchers in many ways[3,5-8].

Rao and Ross pointed out the importance of the cache performance in designing a main memory index and proposed the CSS-Tree(Cache-Sensitive Search Tree) which has a faster search performance than the Binary Search Tree or the T-Tree in the 
read-only OLAP environment[6]. They also proposed the CSB+-Tree which is an extension of the CSS-Tree and can improve the cache performance of the B+-Tree[7].

Sitzmann and Stuckey proposed the pR-Tree(partial R-Tree), which adjusts the size of the R-Tree node to that of cache block and deletes unnecessary information within MBR(Minimum Bounding Rectangle) to store more information in a node[8]. Kim and Cha proposed the CR-Tree(Cache-conscious R-Tree) which compresses MBR of an entry to include more entries in a node[3]. The typical approach for cache performance improvement is to minimize cache misses by reducing the size of the entry to increase the fanout and storing more entries in a node. But, in this approach, the update performance is generally lowered due to additional operations to recover the compressed entry information and cache miss occurring when moving between nodes still results in the lowered performance of the entire system.

In order to solve the above problems, this paper proposes the SPR-Tree(Selective Prefetching R-Tree), an extended R-Tree indexing method, which applies the selective prefetching to the R-Tree in the main memory. The SPR-Tree loads the child node onto the cache memory in advance to extend the size of the node to be optimized for prefetching without transforming the R-Tree radically and reduce cache misses occurring when moving between nodes. The performance improvement of the SPRTree using selective prefetching is in proportion to the size and the number of the nodes to access. Therefore, it is more effective in the range query than in the point query.

The rest of this paper is organized as follows. Chapter 2 introduces selective prefetching and then analyzes the existing cache conscious index methods. Chapter 3 explains the structure of the SPR-Tree and algorithms for the SPR-Tree. In Chapter 4, the performance of the SPR-Tree is analyzed and the results of the SPR-Tree evaluation are presented. Finally, the conclusion is provided in Chapter 5 .

\section{Related Works}

This chapter will introduce selective prefetching and analyze the various existing cache conscious index methods.

\subsection{Selective Prefetching}

The cache memory is used to provide data to the processor in a fast way. Located between the main memory and the processor, the cache memory generally consists of 2 layers; L1 cache and L2 cache. L1 cache is located between the register and L2 cache, while L2 cache is located between L1 cache and the main memory[4]. When the processor is accessing data, if the data is present in the cache memory, it is called "cache hit" and if the data is not present, it is called "cache miss".

The cache block is the basic transfer unit between the cache memory and the main memory. The current systems tend to have bigger size of the cache block and largercapacity of the cache memory. Typical cache block size ranges from 32 bytes to 128 bytes. Generally, the data cache follows the basic principle of the data locality. A tree 
structure has the low data locality as data to refer to is accessed through the pointer. Therefore, in order to improve the cache performance in the tree structure, the amount of data to access should be reduced or selective prefetching should be executed.

The selective prefetching is a technique to selectively load data into the cache memory in advance to accelerate the program execution. Especially, the selective prefetching can reduce cache misses by loading data which does not exist in the cache memory before the processor requests it. In order to reduce cache misses in the RTree, the selective prefetching should be used to reduce memory delay occurring when accessing nodes overall. The selective prefetching is controlled in two ways; the hardware-based prefetching where the prefetching is automatically carried out by the processor and the software-based prefetching where a prefetching command is inserted into the program source code[9].

\subsection{Cache Conscious Index Methods}

The CSB+-Tree is a variant of the $\mathrm{B}+-$ Tree, removing all child node pointers except the first child node pointer to store child nodes consecutively in order to reduce cache misses in the B+-Tree[7]. But, this method of eliminating pointers is not so effective in the R-Tree where pointers account for a relatively small part. And since child nodes are consecutively stored in the CSB+-Tree, every update operation requires reorganization of consecutively arranged child nodes.

The $\mathrm{pR}$-Tree is a variant of the R-Tree, removing child MBR's coordinate values overlapped with those of parent MBR to reduce cache misses in the R-Tree[8]. This method also eliminates the pointers, like in the CSB+-Tree, and shows better performance when the number of entries is small. However, this method has worse performance as the number of entries increases, since the number of child MBR's coordinate values overlapped with those of parent MBR is decreased. In addition, due to the elimination of overlapped child MBR's coordinate values, additional operations are needed for reorganization of the eliminated coordinate values, which lowers the update performance.

The CR-Tree is a kind of the R-Tree that compresses MBR, which accounts for most of indexes, and uses the compressed MBR as a key[3]. In the CR-Tree, MBR is compressed according to the following procedure; MBR of the child node is represented in relative coordinates to MBR of the parent node and it is quantized so that it can be represented in definite bits. However while compressing MBR in the CR-Tree, a small error can occur and this may produce a wrong result(i.e., false hit). Moreover, additional operations for reorganization of the compressed MBR in the update operation can lower the update performance.

\section{SPR-Tree}

This chapter will describe the SPR-Tree, a main memory-based R-Tree using selective prefetching. First, the structure and characteristics of the SPR-Tree will be given and then the algorithms used in the SPR-Tree also will be suggested. 


\subsection{Structure}

The SPR-Tree, similar to the R-Tree, has the root node, the intermediate node, and the leaf node. All operations on the SPR-Tree start from the root node and the references to real data objects exist only in the leaf node. Figure 1 illustrates the node structure of the SPR-Tree. The SPR-Tree uses a rectangle, which is a rectilinear shape that can completely contain other rectangles or data objects.

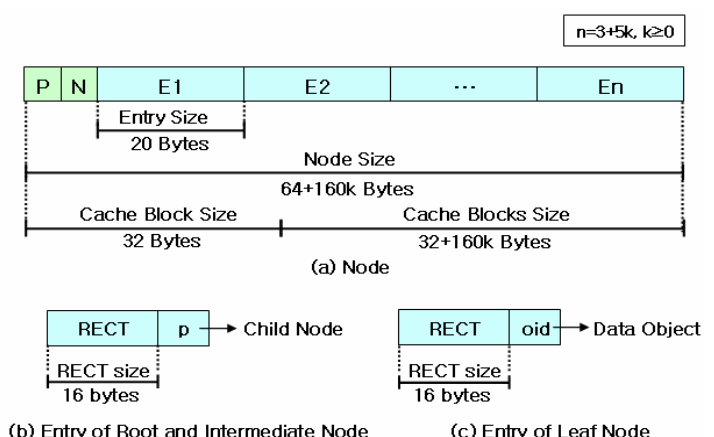

Fig. 1. Node Structure of the SPR-Tree

In Figure 1(a), $P$ and $N$ represent the node level and the number of entries in a node, respectively. Each of $E 1, E 2, \ldots, E n(n=3+5 k, k \geq 0)$ represents an entry which has two types, that is, an entry for the root node or the intermediate node and an entry for the leaf node. Figure 1(b) shows the entry for the root node or the intermediate node, where RECT is a rectangle which completely contains all rectangles in the child node's entries and $p$ is an address of a child node. Figure 1(c) represents the entry for the leaf node, where $R E C T$ is a rectangle which completely contains a data object and oid refers to the data object.

Since the SPR-Tree nodes adjust the number of entries suited to the cache block; the SPR-Tree decides the node size in proportion to the cache block size. Generally, the cache block size can be 32 or 64 bytes. If the cache block size is 32 bytes, the node size becomes $64+160 k(k \geq 0)$ bytes and if it is 64 bytes, the node size becomes $64+320 k(k \geq 0)$.

Figure 2 shows an example of the SPR-Tree. As the Figure 2 shows, rectangles can enclose a single data object or one or more rectangles. For example, rectangle R8, which is at the leaf level of the SPR-Tree, contains data object O. Rectangle R3, which is at the intermediate level of the SPR-Tree, contains rectangles R8, R9, and R13. Rectangle R1, which is at the root level, contains rectangles R3 and R4. In Figure 2 , a prefetching node group enclosed by a dotted line is determined according to the node size.

\subsection{Algorithms}

This section will describe the search, node split insert, and delete algorithms of the SPR-Tree in detail. 


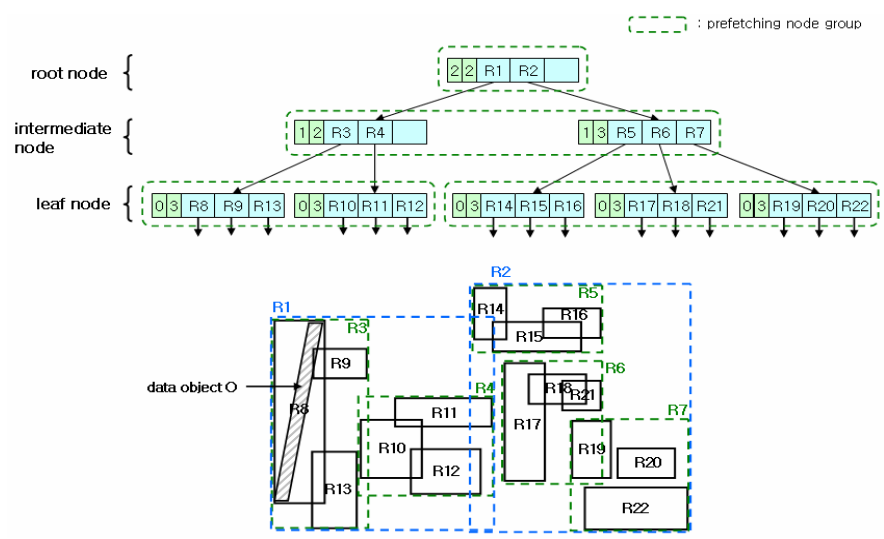

Fig. 2. Example of the SPR-Tree

\subsubsection{Insert Algorithm}

The insert operation repeats, from the root node down to the leaf node, a process of using lower node's rectangle information contained in entries of each node to determine whether the size expansion of the node can be minimized or not when an object is inserted into the leaf node. At this time, if the leaf node becomes full, then a node split occurs. In the insert algorithm of the SPR-Tree, prefetching is carried out while looking for the leaf node to insert an entry. Figure 3 shows the insert algorithm of the SPR-Tree.

\subsubsection{Delete Algorithm}

The delete operation repeats, from the root node down to the leaf node, a process of using lower node's rectangle information contained in entries of each node to determine whether a query region is contained or overlapped in the lower nodes. At this time, if an entry is deleted and the number of remaining entries is below the minimum number of entries in the leaf node, then the leaf node is deleted and its remaining entries are reinserted into the SPR-Tree. The delete algorithm of the SPR-Tree uses a prefetching command based on the node size. The child node to be accessed is prefetched after the current node according to the node size. Figure 4 shows the delete algorithm of the SPR-Tree.

\subsubsection{Search Algorithm}

The search operation descends the SPR-Tree from the root node to the leaf node. And, it repeats a process of using lower node's rectangle information contained in entries of each node to determine whether the lower node contains or overlaps a query region or not. If the lower node is contained or overlapped with the query region, the search operation follows the lower node as the root node until it reaches the leaf node. The search algorithm of the SPR-Tree uses a prefetch command to prefetch a child node to be accessed after the current node. If the node has few entries, the SPR-Tree makes a prefetching node group using some nodes at the same level and prefetches it. While the node has many entries, it prefetches only a child node to be accessed into the cache memory. Figure 5 shows the search algorithm of the SPR-Tree. 


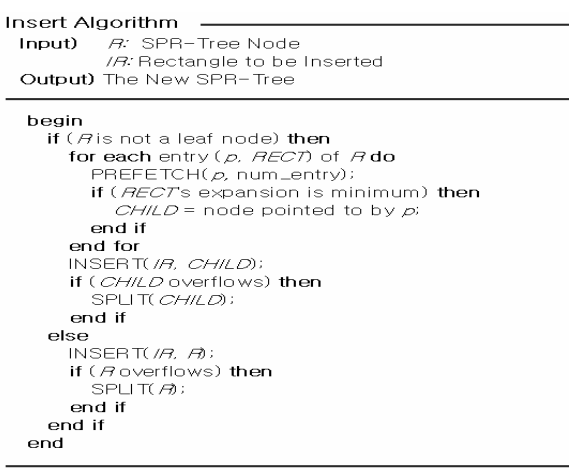

Fig. 3. Insert Algorithm

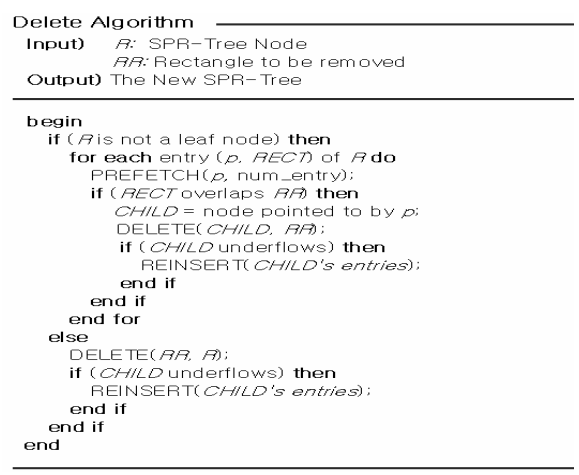

Fig. 4. Delete Algorithm

\subsubsection{Node Split Algorithm}

When a leaf node is full during the execution of an insert operation in the SPR-Tree, the node split operation must be executed. First, the entries in the node are divided into two nodes with minimum rectangle expansion. If the number of entries in the parent node exceeds the maximum number of entries in the parent node due to the node split, the parent node also must be split. The node split algorithm prefetches the current node before split and creates two new nodes to distribute the entries of the current node. Figure 6 shows the node split algorithm of the SPR-Tree.

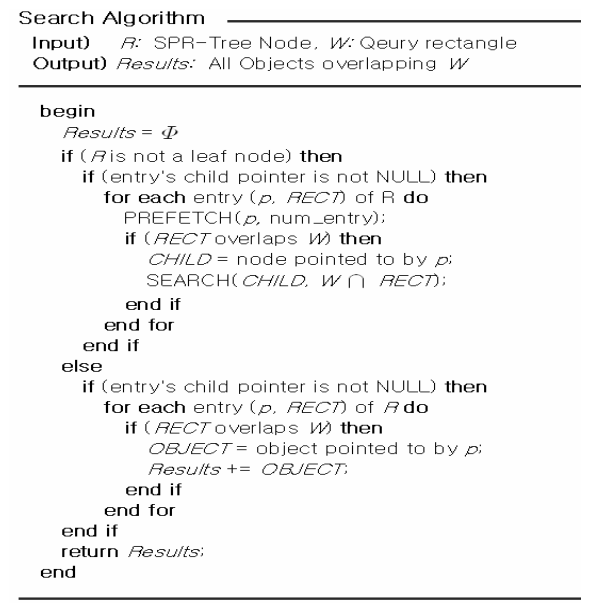

Fig. 5. Search Algorithm

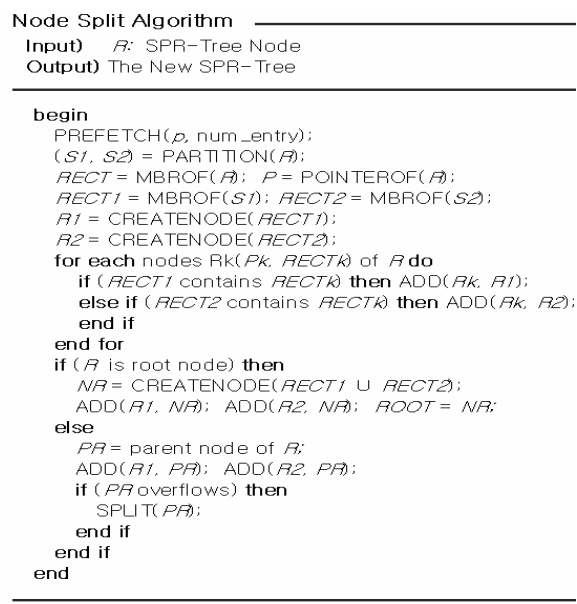

Fig. 6. Node Split Algorithm

\section{Performance Evaluation}

The system used in the performance evaluation was equipped with Intel Pentium III $1 \mathrm{GHz}, 1 \mathrm{~GB}$ main memory, and L1 and L2 caches whose block size is 32 bytes. As a test data, we created 10,000 objects, a square with side length of 0.0001 on the average, uniformly distributed in a square with side length of 1 as the whole area. 
Figure 7 shows the performance results of the search operation. The query region was supposed to occupy 30\% 70\% of the whole area. In Figure 7, the SPR-Tree has better search performance than the R-Tree and improvement through prefetching appears more consistent, as memory delay is reduced while accessing nodes. The search performance of the SPR-Tree was improved up to $35 \%$ over the R-Tree.

Figure 8 shows the performance results of the search operation in a skewed data set. As shown in Figure 8, the larger the node size is, the better search performance it has. This is because there is more reduced memory delay time due to prefetching, as the spatial objects are skewed, which increases overlapping between nodes and the number of nodes to access. The search performance of the SPR-Tree was improved up to $40 \%$ over the R-Tree for skewed data set.

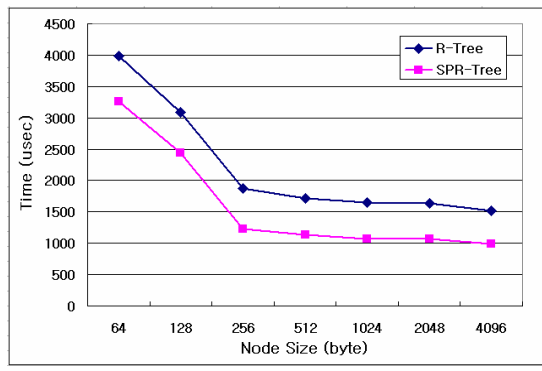

Fig. 7. Performance of Search Operations

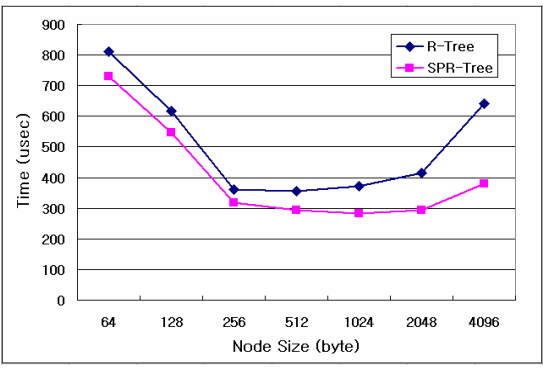

Fig. 8. Performance of Search Operations in Skewed Data Set

Figure 9 shows the performance results of the insert operation. The spatial objects were inserted and the side length of the objects was 0.0001 on the average. As shown in Figure 9, when the node size is larger, the insert time increases, but we can see that the performance improvement rate increases due to prefetching. This is because when prefetching is used, larger node size brings higher performance. The insert performance of the SPR-Tree showed up to $10 \%$ improvement over the R-Tree.

Figure 10 shows the performance results of the delete operation. We deleted objects involved in the region whose side length was 0.001 on the average. In Figure 10, the larger node size generally leads to the better performance of the delete operation and the performance improvement through prefetching is consistent, as memory delay

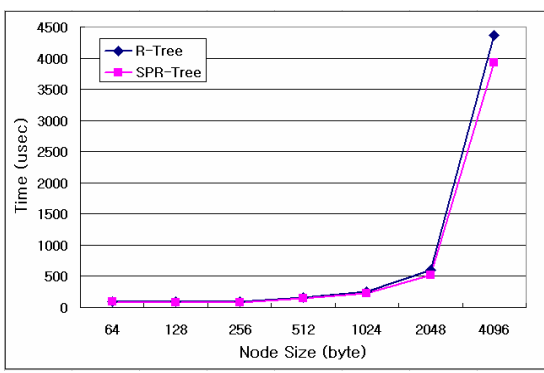

Fig. 9. Performance of Insert Operations

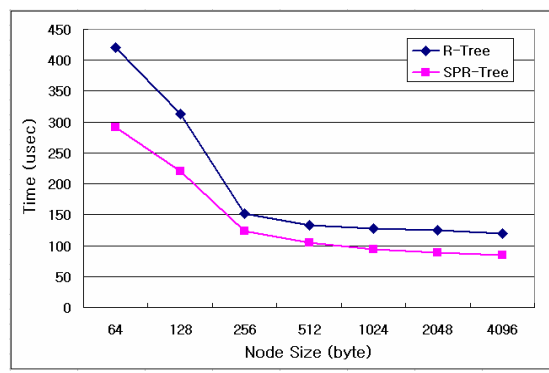

Fig. 10. Performance of Delete Operations 
time reduced by prefetching is consistent while accessing nodes. In the evaluation, the delete performance of the SPR-Tree was improved up to $30 \%$ over the R-Tree.

\section{Conclusion}

Recently an approach that can improve the main memory-based R-Tree index structure by reducing the node size was proposed. However, in this approach, the update performance is lowered due to additional operations to recover the compressed entry information and, still, cache misses occurring when moving between nodes contributes to the lowered performance of the entire system.

In order to solve the above problems, this paper proposes the SPR-Tree which applies the selective prefetching to the R-Tree to reduce cache misses as well as eliminate additional cost in the update operation. The SPR-Tree optimizes the node size for prefetching and minimizes cache misses by prefetching child nodes when moving between nodes. In the performance evaluation, the SPR-Tree was improved up to $40 \%$ in the search operation, up to $10 \%$ in the insert operation, and up to $30 \%$ in the delete operation over the R-Tree.

\section{Acknowledgements}

This research was supported by the MIC(Ministry of Information and Communication), Korea, under the ITRC(Information Technology Research Center) support program supervised by the IITA(Institute of Information Technology Assessment).

\section{References}

1. Chen, S., Gibbons, P. B., Mowry, T. C., Valentin, G.: Fractal Prefetching B+-Trees : Optimizing Both Cache and Disk Performances. Proceedings of ACM SIGMOD Conference (2002) 157-168.

2. Guttman, A.: R-Trees: a Dynamic Index Structure for Spatial Searching. Proceedings of ACM SIGMOD Conference (1984) 47-54.

3. Kim, K. H., Cha, S. K., Kwon, K. J.: Optimizing Multidimensional Index Tree for Main Memory Access. Proceedings of ACM SIGMOD Conference (2001) 139-150.

4. Mowry, T. C., Lam, M. S., Gupta, A.: Design and Evaluation of a Compiler Algorithm for Prefetching. Proceedings of International Conference on Architectural Support for Programming Languages and Operating Systems (1992) 62-73.

5. Park, M. S., Lee, S. H.: A Cache Optimized Multidimensional Index in Disk-Based Environments. IEICE Transactions on Information and Systems, Vol.E88-D (2005) 1940-1947.

6. Rao, J., Ross, K. A.: Cache Conscious Indexing for Decision-Support in Main Memory. Proceedings of International Conference on VLDB (1999) 78-89.

7. Rao, J., Ross, K. A.: Making B+-Trees Cache Conscious in Main Memory. Proceedings of ACM SIGMOD Conference (2000) 475-486.

8. Sitzmann, I., Stuckey, P. J.: Compacting Discriminator Information for Spatial Trees. Proceedings of Australasian Database Conference (2002) 167-176.

9. VanderWiel, S. P., Lilja, D. J.: Data Prefetch Mechanisms. ACM Computing Surveys, Vol.32 (2000) 174-199.

10. Zhou, J., Ross, K. A.: Buffering Accesses of Memory-Resident Index Structures. Proceedings of International Conference on VLDB (2003) 405-416. 